\title{
Drug Utilization Evaluation Study and Dose Adjustment in Patients with Kidney Disease in Tertiary Care Hospital
}

\author{
Stephin V. Mathew", Santhosh Uttangi, Dayana Noble, Manisha Ravi, Stephy K. Mathew, \\ J. S. Venkatesh
}

Department of Pharmacy Practice, SCS College of Phamacy, Harapanahalli, Karnataka, India

Email address:

stephinvmathew1@gmail.com (S. V. Mathew)

${ }^{*}$ Corresponding author

\section{To cite this article:}

Stephin V. Mathew, Santhosh Uttangi, Dayana Noble, Manisha Ravi, Stephy K. Mathew, J. S. Venkatesh. Drug Utilization Evaluation Study and Dose Adjustment in Patients with Kidney Disease in Tertiary Care Hospital. International Journal of Biomedical Engineering and Clinical Science. Vol. 7, No. 3, 2021, pp. 52-64. doi: 10.11648/j.ijbecs.20210703.13

Received: July 25, 2021; Accepted: August 26, 2021; Published: August 31, 2021

\begin{abstract}
INTRODUCTION: Kidney disease is becoming a worldwide public health problem with an increase in incidence and prevalence, poor outcomes and high cost. Rational prescription is necessary in kidney disease patients. These patients are at higher risk of developing drug related problems since they need complex therapeutic regimens that include comorbid conditions like diabetes mellitus, hypertension, coronary artery disease and infection that require frequent monitoring and dosage adjustment. Inappropriate use of medications can increase adverse drug effects, which can be reflected by excessive length of hospital stays, excessive health care utilization and cost. OBJECTIVES: The objective of the study was to assess, evaluate and analyze the prescribing pattern of drugs in kidney disease and their dose adjustments in medicine and emergency department of tertiary care teaching hospital. METHODOLOGY: The study was conducted for a period of 6 months. Ethical clearance was obtained from Institutional Ethical Committee of S C S College of Pharmacy, Harapanahalli. Collected data was analyzed to identify the current prescribing trend and dosage regimen in the management of renal failure patients and to know whether the prescribing rationality was obtained in Medicine and Emergency unit in hospital by using KDIGO guidelines. RESULTS: A total of 140 patients were enrolled in the study according to the inclusion criteria in which 104 were males and 36 were females. $134 \mathrm{CKD}$ cases and 6 AKI cases were found. In the study, 105 (75\%) patients were hypertensive, $62(44.28 \%)$ patients were anemic, 54 (38.57\%) patients were diabetic and dyslipidemia was associated with 21 (15\%) patients. 87 patients were on hemodialysis. On the basis of ATC classification of drugs, cardiovascular system (35.7\%) class of drugs was the commonly prescribed followed by drugs for alimentary tract and metabolism (25.97\%), anti-infective (10.11\%) and blood and blood forming agents (7.97\%). Out of 1028 studied drugs, only 105 (10.21\%) required dose adjustment where 76 (72.38\%) were adjusted and $29(27.61 \%)$ were not adjusted. CONCLUSION: This study illustrates the need for proper dose adjustment and drug utilization pattern in patients with renal failure. Appropriate dosing of antibiotics as well as other drugs, including narrow therapeutic drugs play a vital role in preventing dose related adverse reactions and toxicities. This study will provide an outline for management strategies and will influence the decision making process in clinical practice.
\end{abstract}

Keywords: Kidney Disease, Rational Prescription, KDIGO, Emergency Department, Medicine Department

\section{Introduction}

Renal failure is the worldwide public health problem with an increasing incidence, prevalence, poor outcomes and high cost of treatment due to comorbidities and poly pharmacy. The metabolism and excretion of many drugs and their pharmacologically active metabolites depend on normal renal function. [4] Whereas in patients with kidney dysfunction the renal excretion of parent drug and its metabolites will be impaired, thus reduced or absence of excretion by kidneys in renal failure causes alteration in the pharmacokinetics of drug and thus leading to accumulation of drug metabolites resulting in toxicity. [11]

Renal failure patients are commonly seen with several comorbidities such as hypertension, diabetes mellitus, and coronary artery disease. The presence of these comorbidities has two impact. First it increases the cost of the treatment and 
secondly, it becomes a challenge when it comes to the treatment of renal failure patients. The comorbid conditions also lead to the administration of more medications and thus increases the chance of drug interactions. Because of the use of multiple medications, the renal failure patients are at a higher risk of developing medication dosing errors, which is the most important drug related problem seen in patients with renal impairment [2, 3] If the dosing is inappropriate in patients with renal impairment, it can lead to either toxicity or ineffective therapy. In here particularly the elderly patients are at higher risk due to their age related decline in renal function and use of multiple medications to treat comorbid conditions. [28]

Poly pharmacy is common in renal failure patients, for example, in ESRD patients they undergo haemodialysis and receive many medications with multiple doses per day, so that render them in higher risk of developing drug related problems and non-adherence to treatment, [3] therefore drug dosing in renal insufficiency need to be individualized to optimize therapeutic outcomes and to minimize toxicity. [2]

Some of the frequently prescribed drugs that need their doses to be adjusted in renal failure patients are the following:

1) NSAIDs

2) H2- receptor antagonist

3) Biguanides

4) Anti- tubercular drugs

5) Spironolactone

6) Beta- blockers

7) Ciprofloxacin

8) Aminoglycosides

9) ACE Inhibitors

10)Loop diuretics

11)Metoclopramide

12)Digoxin

Table 1. Other Causes Of Renal Impairment.

\begin{tabular}{ll} 
Pyelonephritis & Inflammation and deterioration of the pyelonephrons due to infection, antigens, or other idiopathic causes. \\
Hypertension & Chronic overloading of the kidney with fluid and electrolytes may lead to kidney insufficiency. \\
Diabetes mellitus & The disturbance of sugar metabolism and acid-base balance may lead to or predispose a patient to degenerative renal disease. \\
Nephrotoxic & Certain drugs taken chronically may cause irreversible kidney damage, eg: aminoglycosides, phenacetin, and heavy metals, such as \\
drugs/metals & mercury and lead. \\
Hypovolemia & Any condition that causes a reduction in renal blood flow will eventually lead to renal ischemia and damage. \\
Nephroallergens & Certain compounds may produce an immune type of sensitivity reaction with nephritic syndrome, eg: quartan malaria nephrotoxic serum. \\
\hline
\end{tabular}

\subsection{Pharmacokinetic Considerations}

Uremic patients may exhibit pharmacokinetic changes in bioavailability, volume of distribution, and clearance. [29] The oral bioavailability of a drug in severe uremia may be decreased as a result of disease- related changes in gastrointestinal motility and $\mathrm{P}^{\mathrm{H}}$ caused by nausea, vomiting and diarrhea. Mesenteric blood flow also altered. However, the oral bioavailability of a drug such as propranolol (which has a high first pass) may be increased in patients with renal impairment as a result of the decrease in first- pass hepatic metabolism. [33] The apparent volume of distribution $\left(\mathrm{V}_{\mathrm{D}}\right)$ depend largely on protein binding in plasma or tissues and total body water. Renal impairment may alter the distribution of the drug as a result of changes in fluid balance, drug protein binding or other factors that may cause changes in the apparent volume of distribution. The plasma protein binding of weak acidic drugs in uremic patients is decreased, whereas the protein binding of weak basic drugs is less affected. The decrease in drug protein binding results in a larger fraction of free drug and an increase in the volume of distribution. However, the net elimination half-life is generally increased as a result of the dominant effect of reduced glomerular filtration. [21] Protein binding of the drug may be further compromised due to the accumulation of metabolites of the drug and accumulation of various biochemical metabolites, such as free fatty acids and urea, which may compete for the protein binding sites for the active drug. Total body clearance of drugs in uremic patients is also reduced by either a decrease in glomerular filtration rate and possibly active tubular secretion or reduced hepatic clearance resulting from a decrease in intrinsic hepatic clearance.

\subsection{Markers Used in the Measurement of Glomerurular Filtration Rate}

Several drugs and endogenous substances have been used as markers to measure GFR. These markers are carried to the kidney by the blood via the renal artery and are filtered at the glomerulus [22]. Several criteria are necessary to use a drug as a marker to measure GFR:

1) The drug must be freely filtered at the glomerulus.

2) The drug must neither be reabsorbed nor actively secreted by the renal tubules.

3) The drug should not be metabolized.

4) The drug should not bind significantly to plasma proteins.

5) The drug should neither have an effect on the filtration rate nor alter renal function.

6) The drug should be nontoxic.

7) The drug may be infused in a sufficient dose to permit simple and accurate quantization in plasma and in urine.

Therefore, the rate at which these drug markers are filtered from the blood into the urine per unit of time reflects the GFR of the kidney. Changes in GFR reflect changes in kidney function that may be diminished in uremic conditions.

\subsection{Important Markers Used in the Measurement of GFR}

\subsubsection{Inulin}

Inulin, a fructose polysaccharide, fulfil most of the criteria listed above and is therefore used as a standard reference for the measurement of GFR. In practice, however, the use of 
inulin involves a time-consuming procedure in which inulin is given by intravenous infusion until a constant steady-state plasma level is obtained. [30, 31] Clearance of inulin may then be measured by the rate of infusion divided by the steady-state plasma inulin concentration. Although this procedure gives an accurate value for GFR, inulin clearance is not used frequently in clinical practice. [20, 29]

\subsubsection{Creatinine}

The clearance of creatinine is used most extensively as a measurement of GFR. Creatinine is an endogenous substance formed from creatinine phosphate during muscle metabolism. Creatinine $[8,7,16]$ production varies with age, weight, and gender of the individual. In humans, creatinine is filtered mainly at the glomerulus, with no tubular Reabsorption. However, a small amount of creatinine may be actively secreted by the renal tubules, and the values of GFR obtained by the creatinine clearance tend to be higher than GFR measured by inulin clearance. Creatinine clearance tends to decrease in the elderly patient. The physiologic changes due to aging may necessitate special considerations in administering drugs in the elderly.

\subsubsection{Blood Urea Nitrogen}

Measurement of Blood Urea Nitrogen (BUN) is a commonly used clinical diagnostic laboratory test for renal disease. Urea is the end product of protein catabolism and is excreted through the kidney. [2] Normal BUN levels range from 10 to $20 \mathrm{mg} / \mathrm{dl}$. Higher BUN levels generally indicate the presence of renal disease. [6, 9, 23] However, other factors, such as excessive protein intake, reduced renal blood flow, hemorrhagic shock, or gastric bleeding, may affect increased BUN levels. [17, 23] The renal clearance of urea is by glomerular filtration and partial Reabsorption in the renal tubules. Therefore, the renal clearance of urea is less than creatinine or inulin clearance and does not give a quantitative measure of kidney function.

Table 2. Properties of The Most Commonly Used Biomarkers For Measuring Or Estimating Gfr.

\begin{tabular}{|c|c|c|c|}
\hline FILTRATION MARKER & SOURCE & TYPE & FILTRATION PROPERTY \\
\hline Creatinine & Endogenous & Metabolized from creatinine & $\begin{array}{l}\text { Freely filtered through the glomeruli. } \\
\text { Secreted from the proximal tubule. } \\
\text { Extra renal elimination (stools or sweat) }\end{array}$ \\
\hline Cystatin & Endogenous & Proteinase inhibitor & $\begin{array}{l}\text { Stable plasma concentration. } \\
\text { Freely filtered through glomeruli. } \\
\text { Reabsorbed and metabolized in the proximal tubule. }\end{array}$ \\
\hline Inulin & Exogenous & Fructose polysaccharide & $\begin{array}{l}\text { Physiologically inert. } \\
\text { Freely filtered through the glomeruli. } \\
\text { Not absorbed, secreted, synthesized or metabolized in the kidney. } \\
\text { Stable plasma concentration. }\end{array}$ \\
\hline Iohexol & Exogenous & $\begin{array}{l}\text { Non-ionic water soluble } \\
\text { contrast medium }\end{array}$ & $\begin{array}{l}\text { Freely filtered through glomeruli. } \\
\text { Not secreted or reabsorbed in the tubules }\end{array}$ \\
\hline
\end{tabular}

Table 3. 2012 Kdigo Chronic Kidney Disease Categories Based On Egfr

\begin{tabular}{lll}
\hline CKD CATEGORY & eGFR $\left(\mathbf{m l} / \mathbf{m i n} / \mathbf{1 . 7 3} \mathbf{~ m}^{\mathbf{2}}\right)$ & TERM \\
\hline G1 & $>90$ & Normal \\
G2 & $60-89$ & Mildly decreased \\
G3a & $45-59$ & Mild to moderately decreased \\
G3b & $30-44$ & Moderate to severely decreased \\
G4 & $15-29$ & Severely decreased \\
G5 & $<15$ & Kidney failure \\
\hline
\end{tabular}

\subsubsection{Different Methods of Extracorporeal Removal of Drugs}

\section{(i) Peritoneal Dialysis}

Peritoneal dialysis uses the peritoneal membrane in the abdomen as the filter. The peritoneum consists of visceral and parietal components. [10] The peritoneum membrane provides a large natural surface area for diffusion of approximately $1-2 \mathrm{~m} 2$ in adults; it is permeable to solutes of molecular weights $\leq 30,000$ Da. [15] However, only a small portion of the total splanchnic blood flow $(70 \mathrm{~mL} / \mathrm{min}$ out of $1200 \mathrm{~mL} / \mathrm{min}$ at rest) comes into contact with the peritoneum and gets dialyzed. The dialysis fluid is pumped into the peritoneal cavity, where waste metabolites in the body fluid are discharged rapidly. The dialysate is drained and fresh dialysate is reinstalled and then drained periodically.
Peritoneal dialysis is also more amenable to self-treatment. However, slower drug clearance rates are obtained with peritoneal dialysis compared to hemodialysis, and thus longer dialysis time is required. Many diabetic patients become uremic as a result of lack of control of their disease. About 2 $\mathrm{L}$ of dialysis fluid is instilled into the peritoneal cavity of the patient through a surgically placed resident catheter. The objective is to remove accumulated urea and other metabolic waste in the body. $[12,30]$ The catheter is sealed and the patient is able to continue in an ambulatory mode. Every 4-6 hours, the fluid is emptied from the peritoneal cavity and replaced with fresh dialysis fluid. $[8,9]$

\section{(ii) Haemodialysis}

Haemodialysis uses a dialysis machine and filters blood through an artificial membrane. Haemodialysis requires access to the blood vessels to allow the blood to flow to the 
dialysis machine and back to the body. For temporary access, a shunt is created in the arm, with one tube inserted into an artery and another tube inserted into a vein. The tubes are joined above the skin. For permanent access to the blood vessels, an arteriovenous fistula or graft is created by a surgical procedure to allow access to the artery and vein. Patients who are on chronic haemodialysis treatment need to be aware of the need for infection control of the surgical site of the fistula. At the start of the haemodialysis procedure, an arterial needle allows the blood to flow to the dialysis machine, and blood is returned to the patient to the venous side. Heparin is used to prevent blood clotting during the dialysis period. [1, 5] During haemodialysis, the blood flows through the dialysis machine, where the waste material is removed from the blood by diffusion through an artificial membrane before the blood is returned to the body. Haemodialysis is a much more effective method of drug removal and is preferred in situations when rapid removal of the drug from the body is important, as in overdose or poisoning. In practice, haemodialysis is most often used for patients with end-stage renal failure. [18, 19, 27]

\section{(iii) Hemoperfusion}

Hemoperfusion is the process of removing drug by passing the blood from the patient through an adsorbent material and back to the patient. [24, 26] Hemoperfusion is a useful procedure for rapid drug removal in accidental poisoning and drug overdose. Because the drug molecules in the blood are in direct contact with the adsorbent material, any molecule that has great affinity for the adsorbent material will be removed.

The important factors for drug removal by Hemoperfusion include affinity of the drug for the adsorbent, surface area of the adsorbent, absorptive capacity of the adsorbent, rate of blood flow through the adsorbent, and the equilibration rate of the drug from the peripheral tissue into the blood. $[13,14,16]$

\section{(iv) Hemofiltration}

An alternative to hemodialysis and hemoperfusion is hemofiltration. Hemofiltration is a process by which fluids, electrolytes, and small-molecular-weight substances are removed from the blood by means of low-pressure flow through hollow artificial fibers or flat-plate membranes. Because fluid is also filtered out of the plasma during hemofiltration, replacement fluid is administered to the patient for volume replacement. Hemofiltration is a slow, continuous filtration process that removes non protein-bound small molecules $(<10,000 \mathrm{Da})$ from the blood by convective mass transport. [7, 6] The clearance of the drug depends on the sieving coefficient and ultra-filtration rate. Hemofiltration provides a creatinine clearance of approximately $10 \mathrm{~mL} / \mathrm{min}$ and may have limited use for drugs that are widely distributed in the body, such as aminoglycosides, cephalosporin's, and acyclovir. A major problem with this method is the formation of blood clots within the hollow filter fibers.

It is necessary that kidney function is assessed correctly and the drug doses are adjusted according to the kidney function, [4] to describe the above issues a prospective crosssectional study was planned to assess the dosage regimen and drug utilization evaluation pattern of drugs in renal failure patients at tertiary care hospital.

\section{Objectives of the Study}

\subsection{Primary Objective}

To analyse current prescribing trends and dosage regimen in the management of renal failure patients and to know whether the prescribing rationality was obtained in a study conducted in the tertiary care hospital.

\subsection{Secondary Objective}

1) To make sure quality medication are given to the patient with the decrease in cost of treatment.

2) To create awareness regarding the medications among physicians and patients.

3) To prevent drug related problems and to improve adherence of patients to medications.

\section{Methodology}

\subsection{Source of Data}

Data will be collected from prospective series of prescription of patients who are admitted in the medical ward.

\subsection{Study Site}

Chigateri District Hospital (tertiary care teaching hospital) Davangere.

\subsection{Duration of Study}

The study will be conducted for a period of 6 months.

\subsection{Study Design}

Prospective observational study (subjects are followed to observe future outcome).

\subsection{Study Size}

More than 100 case sheets of patients admitted in medicine department of the hospital.

\subsection{Study Criteria}

The study will be carried out by considering the following inclusion and exclusion criteria.

\subsubsection{Inclusion Criteria}

1) Patient of either sex

2) Patient of age above 18 years

3) Newly detected and known case of kidney diseases

4) Cases with comorbid conditions

5) Diagnosed by the clinician to have kidney disease and currently on drug treatment.

6) Patient admitted for more than 2 days. 


\subsubsection{Exclusion Criteria}

1) Patients who are treated from outpatient department and who do not require hospital stay.

2) Pregnant and lactating women.

3) Pediatric patients.

4) In case of surgical conditions like kidney stone.

5) Conditions other than CKD and AKI conditions.

6) Patients with tumor and trauma.

7) Terminally ill patient who is not in a position to be interviewed.

8) Patient with missing and insufficient data.

\subsection{Materials Used}

1) Patient case sheet

2) Patient profile form

3) Lexicomp (a drug information software)

4) Medscape interaction checker (multiple drug interaction checker)

\subsection{Ethical Consideration}

The ethical clearance for the study was obtained from the institutional ethical committee of SCS College of pharmacy.

\subsection{Study Procedure}

1) A prospective observational study will be conducted in the inpatients in medical ward on DUE and dosage adjustment on renal failure patients in Chigateri General Hospital, Davangare.

2) The data required for the study will be collected from the patient case sheets.

3) Inpatients in medicine ward meeting the inclusion criteria will be enrolled in the study.

4) The demographic details, dose and frequency, both during admission and discharge will be recorded in a properly designed data entry form.

5) All prescriptions meeting the inclusion criteria will be assessed for rationality.

\subsection{Statistical Method}

Data will be represented graphically and analyzed using statistical method like MS Excel.

\section{Results}

A total of 140 patients assessed were found eligible as per our inclusion criteria collected cases were included in the suitable data collection form and drug utilization evaluation studies were conducted in patients with renal impairment, the following results were obtained in the study.

\subsection{Gender Wise Distribution of Patients}

The study showed that the incidence of kidney disease were more in males.

Table 4. Gender wise distribution of patients.

\begin{tabular}{lll}
\hline Gender & Number of patients & Percentage \\
\hline Male & 104 & $74.28 \%$ \\
Female & 36 & $25.21 \%$ \\
Total & 140 & $100 \%$ \\
\hline
\end{tabular}

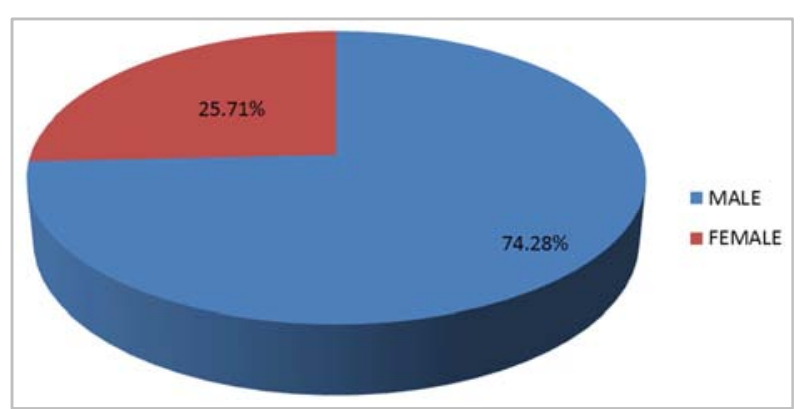

Figure 1. Gender wise distribution of patients.

\subsection{Age Wise Distribution of Patients}

A total of 140 patients were enrolled into different groups in which majority of patients belongs to the age groups of 4150 years $(33.57 \%)$ and $51-60$ years $(33.57 \%)$ followed by $61-$ 70 years $(10.71 \%)$.

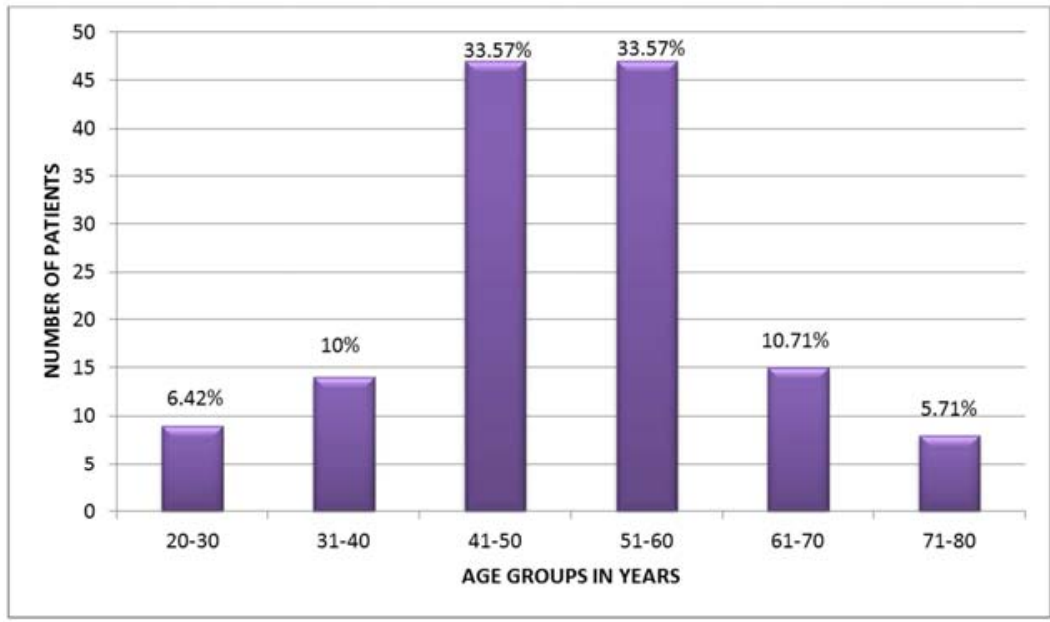

Figure 2. Age wise distribution of patients. 
Table 5. Age wise distribution of patients.

\begin{tabular}{lll}
\hline Age groups & Number of patients & Percentage \\
\hline $20-30$ & 9 & $6.42 \%$ \\
$31-40$ & 14 & $10 \%$ \\
$41-50$ & 47 & $33.57 \%$ \\
$51-60$ & 47 & $33.57 \%$ \\
$61-70$ & 15 & $10.71 \%$ \\
$71-80$ & 8 & $5.71 \%$ \\
\hline
\end{tabular}

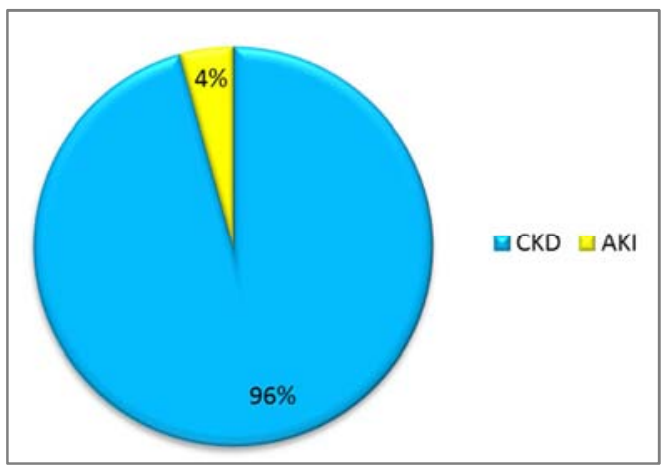

Figure 3. Types of Renal Disease.

\subsection{Types of Renal Disease}

Among 140 patients, $95.7 \%$ patients were diagnosed as CKD and $4.48 \%$ as AKI.

Table 6. Types of Renal Disease.

\begin{tabular}{lll}
\hline Types of Renal Disease & No of patients & Percentage \\
\hline CKD & 134 & $95.7 \%$ \\
AKI & 6 & $4.48 \%$ \\
\hline
\end{tabular}

\subsection{Stages of CKD}

Out of 140 patients most of patients belonged to stage 5 $(41.42 \%)$ followed by stage 4 (32.83\%).

Table 7. Stages of CKD.

\begin{tabular}{lll}
\hline Stages & No. of Patients & Percentage \\
\hline I & 6 & $4.47 \%$ \\
II & 7 & $5.22 \%$ \\
III & 19 & $14.179 \%$ \\
IV & 44 & $32.83 \%$ \\
V & 58 & 41.48 \\
\hline
\end{tabular}

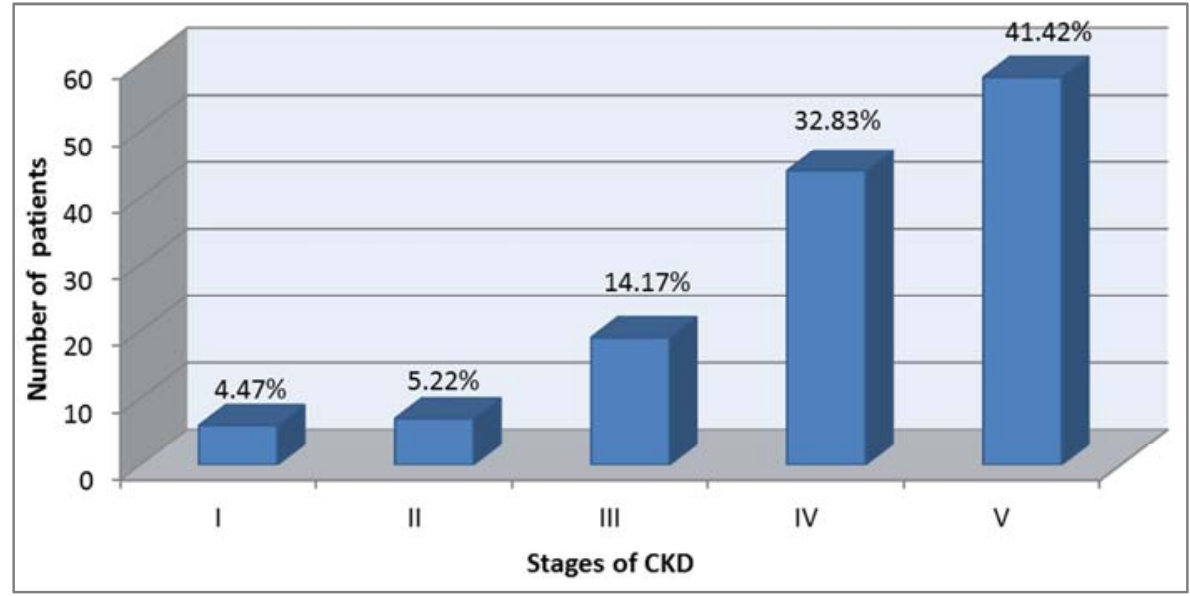

Figure 4. Stages of CKD.

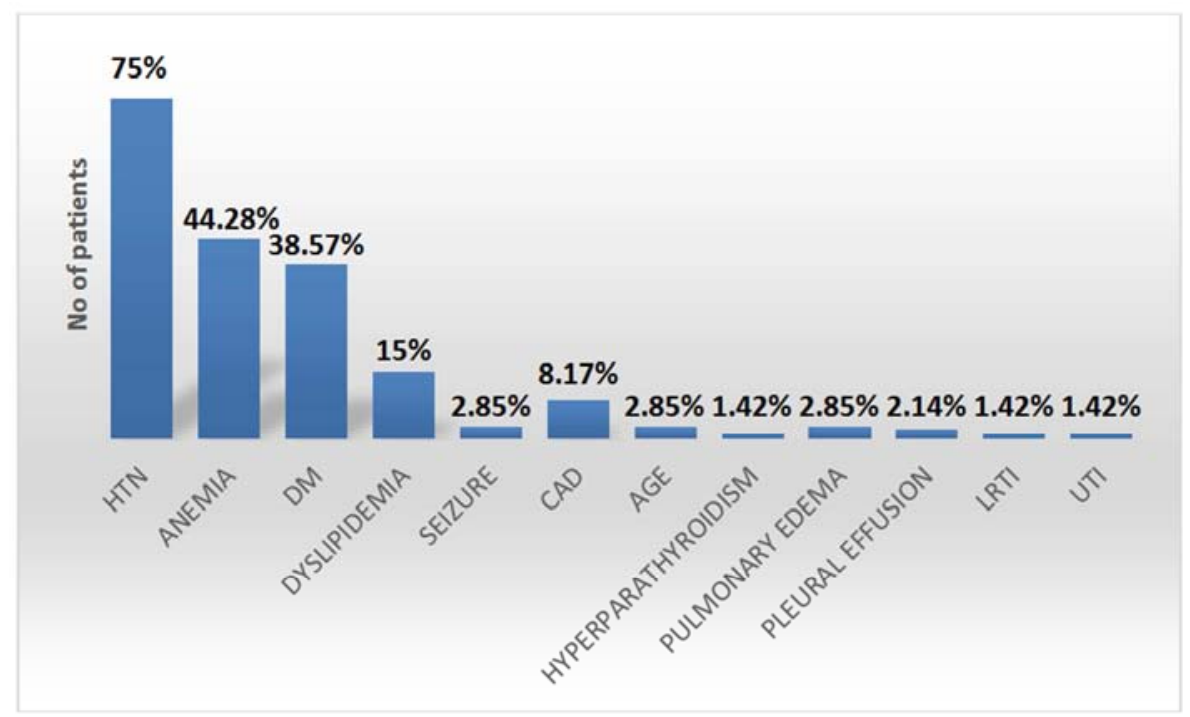

Figure 5. Comorbid conditions. 


\subsection{Comorbid Conditions Associated with the Study}

All patients had same or other comorbid illnesses. The below figure depicts the distribution of most common associated diseases prevailing in CKD patients.

Table 8. Comorbid conditions.

\begin{tabular}{lll}
\hline Disease & No. of patients & Percentage \\
\hline Hypertension & 105 & $75 \%$ \\
Anemia & 62 & $44.28 \%$ \\
Diabetes mellitus & 54 & $38.57 \%$ \\
Dyslipidemia & 21 & $15 \%$ \\
Seizure & 4 & $2.85 \%$ \\
CAD & 12 & $8.17 \%$ \\
AGE & 4 & $2.85 \%$ \\
Hyperparathyroidism & 2 & $1.42 \%$ \\
Pulmonary edema & 4 & $2.85 \%$ \\
Pleural effusion & 3 & $2.14 \%$ \\
LRTI & 2 & $1.42 \%$ \\
UTI & 2 & $1.42 \%$ \\
\hline
\end{tabular}

No of Patients in Hemodialysis
$62.14 \%$ patients were found to be in hemodialysis while $37.58 \%$ patients were not in hemodialysis

Table 9. No. of Patients in Hemodialysis.

\begin{tabular}{lll}
\hline Dialysis & No of patients & Percentage \\
\hline Yes & 87 & $62.14 \%$ \\
No & 53 & $37.85 \%$ \\
\hline
\end{tabular}

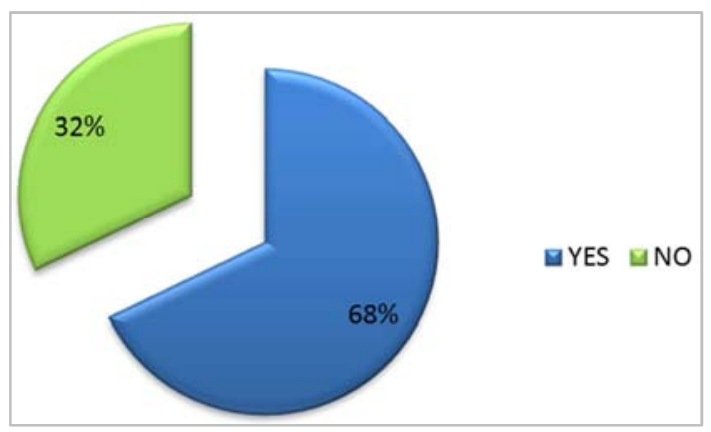

Figure 6. No. of Patients in Hemodialysis.

\subsection{Prescription Analysis}

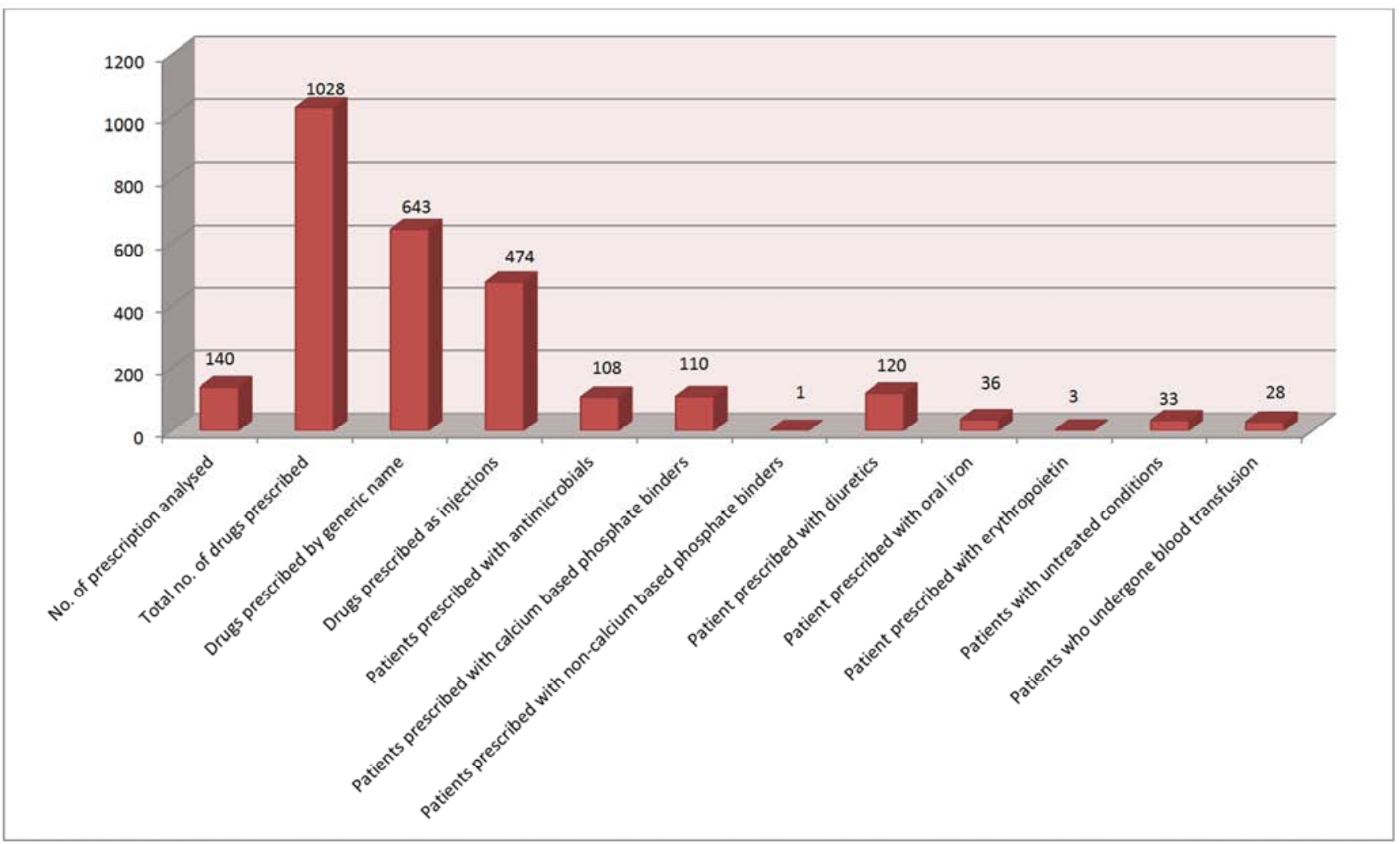

Figure 7. Prescription analysis.

Table 10. Prescription analysis.

\begin{tabular}{ll}
\hline Prescription details & Number (\%) \\
\hline No. of prescription analysed & 140 \\
Total no. of drugs prescribed & 1028 \\
Drugs prescribed by generic name & $643(62.54 \%)$ \\
Drugs prescribed as injections & $474(46.10 \%)$ \\
\hline
\end{tabular}




\begin{tabular}{ll}
\hline Prescription details & Number (\%) \\
\hline Patients prescribed with antimicrobials & $108(77.14 \%)$ \\
Patients prescribed with calcium based phosphate binders & $110(78.57 \%)$ \\
Patients prescribed with non-calcium based phosphate binders & $1(0.71 \%)$ \\
Patient prescribed with diuretics & $120(85.71 \%)$ \\
Patient prescribed with oral iron & $36(26.71 \%)$ \\
Patient prescribed with erythropoietin & $3(2.14 \%)$ \\
Patients with untreated conditions & $33(23.57 \%)$ \\
Patients who undergone blood transfusion & $28(20 \%)$ \\
No. of contraindicated drug prescribed & $21(2.04 \%)$ \\
\hline
\end{tabular}

\subsection{Drug Utilization Pattern}

A total of 140 prescriptions were analysed. The below table shows the observations.

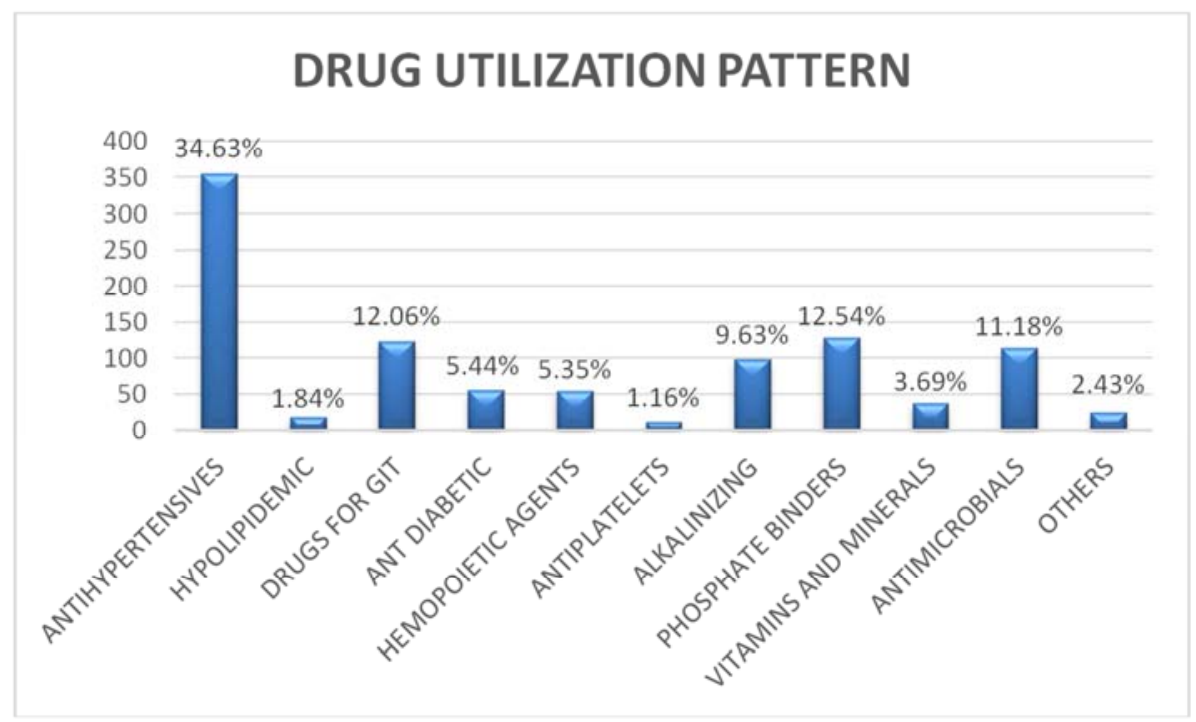

Figure 8. Drug utilization pattern.

Table 11. Drug utilization pattern.

\begin{tabular}{lll}
\hline Drug class & Number & Percentage \\
\hline Antihypertensives & 356 & $34.63 \%$ \\
CCB & 94 & $9.14 \%$ \\
Diuretics & 129 & $12.54 \%$ \\
ACE inhibitors & 8 & $0.77 \%$ \\
Beta blockers & 25 & $2.43 \%$ \\
Clonidine & 82 & $7.97 \%$ \\
ARB & 7 & $0.68 \%$ \\
Hypolipidemic agents & 19 & 1.84 \\
Statins & 10 & $0.97 \%$ \\
Fibrins & 1 & $0.097 \%$ \\
Statin + Aspirin & 8 & $0.77 \%$ \\
Drugs for GIT & 124 & $12.06 \%$ \\
H2 blockers & 67 & $6.51 \%$ \\
PPIs & 57 & $5.54 \%$ \\
Anti -diabetic drugs & 56 & $5.44 \%$ \\
Insulin & 30 & $2.91 \%$ \\
Oral hypoglycemic & 26 & $2.52 \%$ \\
Haemopoietic Agents & 55 & $5.35 \%$ \\
Iron oral & 36 & $3.50 \%$ \\
Folic acid & 16 & $1.55 \%$ \\
Erythropoietin & 3 & $0.29 \%$ \\
Anti -platelet Agents & 12 & $1.16 \%$ \\
Aspirin & 7 & $0.68 \%$ \\
Aspirin + clopidogrel & 5 & $0.48 \%$ \\
Alkalinising Agent & 99 & $9.63 \%$ \\
Phosphate Binders & 129 & $12.54 \%$ \\
Calcium Carbonate & 70 & $6.80 \%$ \\
\hline & & \\
\hline
\end{tabular}




\begin{tabular}{lll}
\hline Drug class & Number & Percentage \\
\hline Calcium Acetate & 59 & $5.73 \%$ \\
Vitamins and Minerals & 38 & $3.69 \%$ \\
Vitamin B12 & 11 & $1.07 \%$ \\
Vitamin D & 27 & $2.62 \%$ \\
Antimicrobials & 115 & $11.18 \%$ \\
Others & 25 & $2.43 \%$ \\
\hline
\end{tabular}

\subsection{Drugs Classified According to ATC Classification}

Table 12. Drugs classified according to ATC classification.

\begin{tabular}{llll}
\hline$\#$ & Class of medicine & Number & Percentage \\
\hline A & Alimentary tract and metabolism & 267 & $25.97 \%$ \\
B & Blood and blood forming organs & 82 & $7.97 \%$ \\
C & Cardiovascular system & 367 & $35.7 \%$ \\
D & Dermatology system & 0 & $0 \%$ \\
G & Genito-urinary system and sex hormones & 63 & $6.12 \%$ \\
H & Systemic hormonal preparations & 24 & $2.33 \%$ \\
J & Anti-infective for systemic use & 104 & $10.11 \%$ \\
L & Antineoplastic and immunomodulating agents & 0 & $0 \%$ \\
M & Musculo-skeletal system & 11 & $1.07 \%$ \\
N & Nervous system & 36 & $3.5 \%$ \\
P & Anti-parasitic products, insecticides and repellents & 0 & $0 \%$ \\
R & Respiratory system & 5 & $0.48 \%$ \\
S & Sensory organs & 0 & $0 \%$ \\
V & Various & 70 & $6.80 \%$ \\
\hline
\end{tabular}

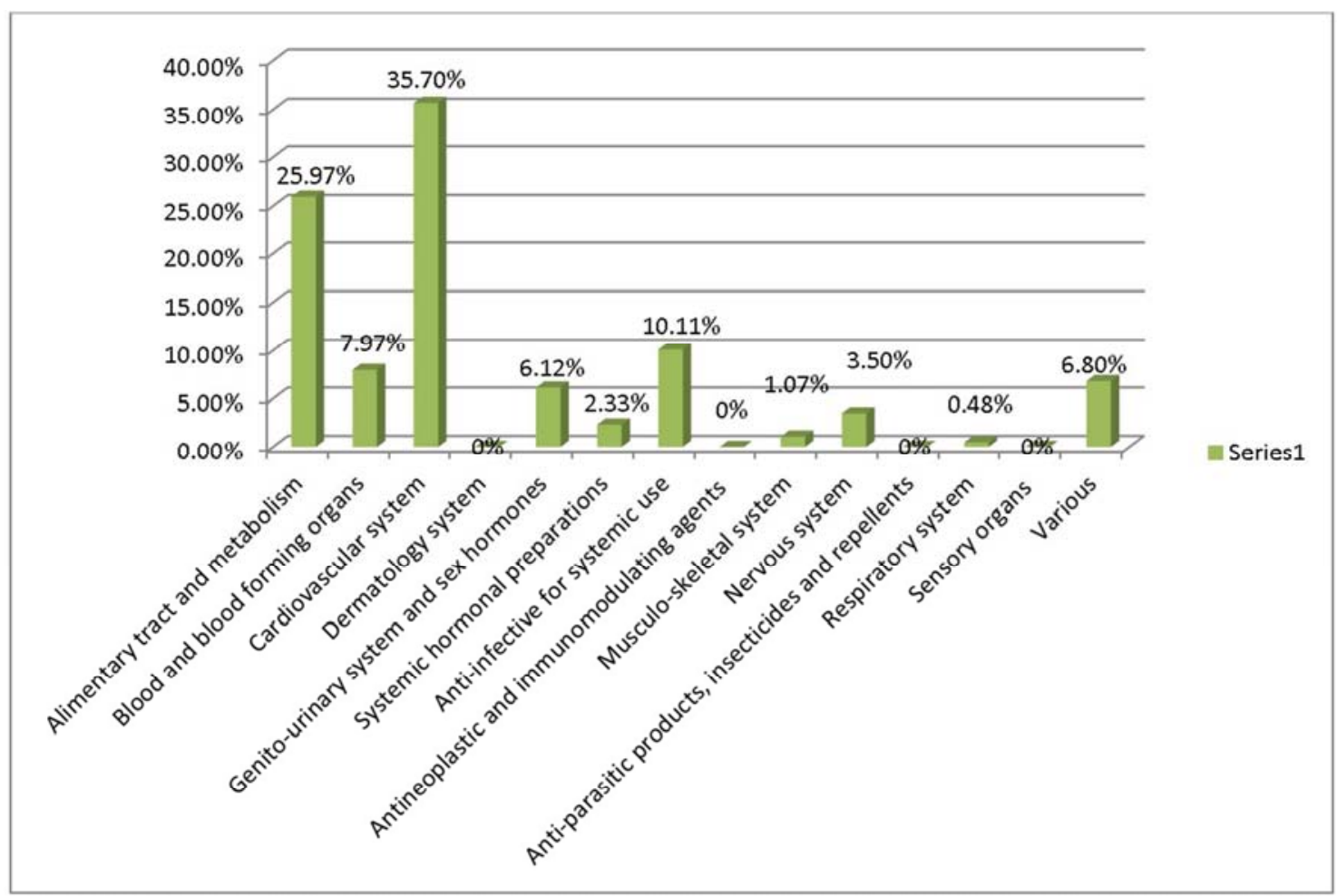

Figure 9. Drugs classified according to ATC classification.

\subsection{No of Prescription That Require Dose Adjustment}

Most of the prescription required dose adjustment (67.85\%).

Table 13. Prescription require dose adjustment.

\begin{tabular}{lll}
\hline Cases & No of prescription & Percentage \\
\hline Prescription required dose adjustment & 95 & $67.85 \%$ \\
Prescription does not require dose adjustment & 45 & $32.14 \%$ \\
\hline
\end{tabular}




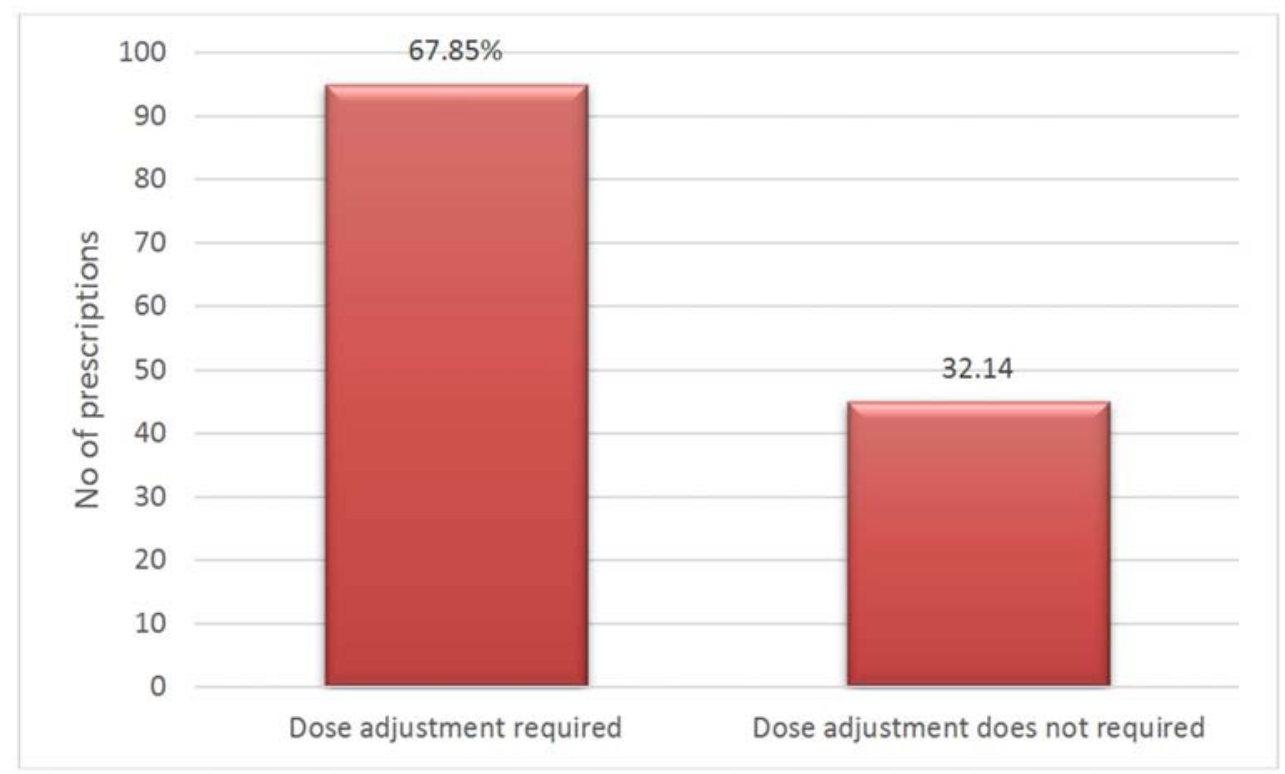

Figure 10. Prescription require dose adjustment.

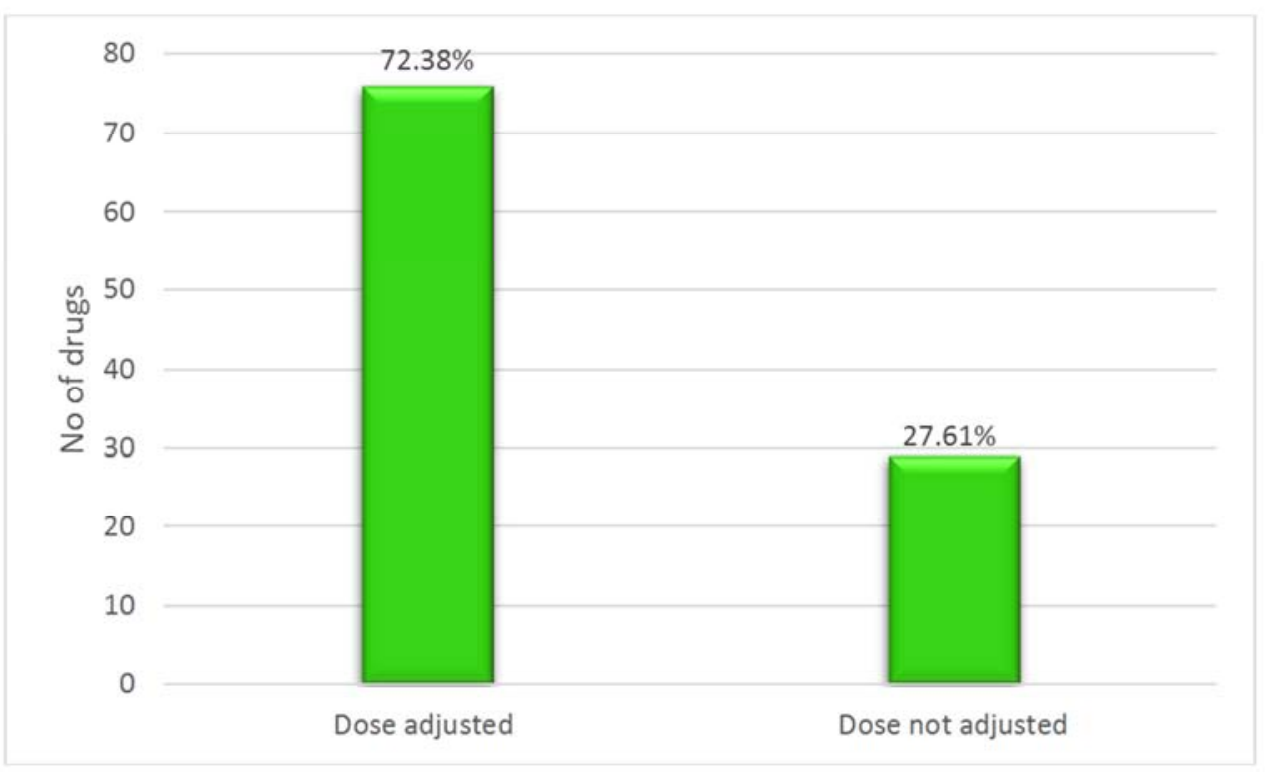

Figure 11. Dose adjusted and non-adjusted drugs in patients with renal impairment.

\subsection{Dose Adjusted and Non-adjusted Drugs in Patients with Renal Impairment}

A total of 1028 drugs analysed 105 drugs were needed to be dose adjusted among them 76 drugs were properly adjusted and 29 drugs were not adjusted.

Table 14. Dose adjusted and non-adjusted drugs in patients with renal impairment.

\begin{tabular}{lll}
\hline Drugs & No of drugs & Percentage \\
\hline Drugs dose adjusted & 76 & $72.38 \%$ \\
Drugs not dose adjusted & 29 & $27.61 \%$ \\
Total & 105 & $100 \%$ \\
\hline
\end{tabular}

\section{Discussion}

Kidney disease is becoming a worldwide public health problem with an increase in incidence and prevalence, poor outcomes and high cost $[32,21]$. Many drugs are extensively excreted by the kidney and therefore they will accumulate in patients with poor renal function if not properly administered. Kidney disease can even lead to other health problem including weak bones, nerve damage and malnutrition. [27]

The study was a prospective observational for a period of 6 months. A total of 140 patients were included in the study according to the inclusion criteria. In 140 patients 104 (74.28\%) were males and $36(25.71 \%)$ were females.

The demographic reports of our study showed high incidence of renal impairment in males 104 (74.28) over females 36 (25.71\%) which were similar results with epidemiological studies conducted by Sindhura $\mathrm{P}$ et al in assessment of drug prescribing pattern in patients of CKD along with CVD where the domination of males $(73.3 \%)$ 
over females $(26.6 \%)$.

The incidence of renal impairment in our study was most seen in the age groups $41-50$ years and 51-60 years (33.57\%). This is similar to the study conducted by Divya Agrawal et al, in which the disease was commonly seen in the age group 41-50 years.

Out of 140 patients in our study, most patients belonged to stage V of CKD (41.42\%) followed by stage IV (32.83\%) of CKD. Most of the patients were first diagnosed in an advanced stage of CKD. This was similar in the study conducted by Rajiv Ahlawat et al on drug utilization pattern in CKD patients in which most of the patient belonged to stage $\mathrm{V}(42 \%)$.

In our study, $105(75 \%)$ patients were hypertensive, 62 (44.28\%) patients were anemic, $54(38.57 \%)$ patients were diabetic and dyslipidemia was associated with $21(15 \%)$ patients. Almost similar results showed in Divya Agrawal et al in the study of drug utilization pattern in CKD with emphasis on antibiotics showed 175 (95\%) patients were hypertensive, $161(87 \%)$ patients were diabetic, $159(86 \%)$ patients were anemic. $83 \%$ were associated with dyslipidemia.

Among 140 patients in our study, 87 (62.14\%) were found to be on hemodialysis. However, the study by Rajiv Ahlawat et al reported only $18 \%$ of the patients on hemodialysis.

In our study, the most commonly prescribed class of drugs were anti-hypertensives $(34.63 \%)$, followed by phosphate binders $(12.56 \%)$, drugs used for GIT $(12.06 \%)$, antimicrobials $(11.18 \%)$, alkalinizing agents $(9.63 \%)$. Among anti-hypertensive, diuretics was the most prescribed followed by calcium channel blockers. The study conducted by Rajiv Alhawat et al found anti-hypertensive drugs $(28.8 \%)$ as the most prescribed drugs followed by hematopoietic drugs $(15.2 \%)$, phosphate binders $(14.3 \%)$ and vitamins and minerals (13.3\%).

On the basis of ATC classification of drugs, cardiovascular system (35.7\%) class of drugs was the commonly prescribed followed by drugs for alimentary tract and metabolism (25.97\%), anti-infective (10.11\%) and blood and blood forming agents (7.97\%). Almost similar results were obtained in the study conducted by Sindhura $\mathrm{P}$ et al in assessment of drug prescribing pattern in patients of CKD with CVD in which the drugs for alimentary tract and metabolism $(28.93 \%)$ were mostly prescribed followed by cardiovascular system drugs (23.47\%) and blood and blood forming agents (18.96\%).

Out of 1028 studied drugs, only 105 (10.21\%) required dose adjustment where $76(72.38 \%)$ were adjusted and 29 (27.61\%) were not adjusted. [13, 19] However, the study by Abdulrahman M Alahat et al on evaluation of applying drug dose adjustment by physicians in patients with renal impairment showed out of 502 studied drugs, 39\% required dose adjustment and $46.9 \%$ were adjusted while $53.1 \%$ were not adjusted.

\section{Conclusion}

Chronic kidney disease, also called chronic kidney failure, describes the gradual loss of kidney function. Its symptoms include weight loss and poor appetite, swollen ankles, feet or hands as a result of water retention (edema), shortness of breath, tiredness, blood in urine etc. Kidneys can become damaged from physical injury or a disease like diabetes, high blood pressure, or other disorders. From our study, the following conclusions can be made;

1) This study analyzed a total of 140 patients with kidney disease. Male patients having more renal disease than females.

2) Renal disease was more commonly seen in the age group 41-50 years and 51-60 years.

3) Most patients belonged to stage V of CKD (41.42\%) followed by stage IV (32.83\%) of CKD.

4) 87 patients were found to be on hemodialysis.

5) The most commonly prescribed class of drugs were anti-hypertensives $(34.63 \%)$, followed by phosphate binders $(12.56 \%)$, drugs used for GIT (12.06\%), antimicrobials (11.18\%), alkalinizing agents $(9.63 \%)$.

6) On the basis of ATC classification of drugs, cardiovascular system (35.7\%) class of drugs was the commonly prescribed followed by drugs for alimentary tract and metabolism (25.97\%), anti-infective (10.11\%) and blood and blood forming agents (7.97\%).

7) Among 140 prescriptions, 95 prescription require dose adjustment while 45 prescriptions do not require dose adjustment.

8) Out of 1028 studied drugs, only 105 required dose adjustment where 76 were adjusted and 29 were not adjusted.

A high number of medications are prescribed for patients with kidney disease due to their comorbid conditions and thereby we can find an increment in the number of drug-drug interaction as well as adverse events. This study illustrates the need for proper dose adjustment and drug utilization pattern in patients with renal failure. Appropriate dosing of antibiotics as well as other drugs, including narrow therapeutic drugs play a vital role in preventing dose related adverse reactions and toxicities. This study will provide an outline for management strategies and will influence the decision making process in clinical practice.

\section{Strength and Limitations}

\subsection{Strengths of the Study}

1) Better involvement of clinical pharmacist in emergency and medicine department.

2) Created awareness regarding the medications among physicians and patients.

3) Data from this study can be used for various pharmacoepidemiological studies (cohort).

4) The result from this study could be useful to prevent drug related problems and to improve adherence to patients to medications. 


\subsection{Limitations of the Study}

1) Duration of the study was only for 6 months.

2) Illegible hand writing in medical records.

3) Incomplete information on patient medication history.

4) Lack of patient compliance.

\section{References}

[1] Tamilselvan T, Veerapandiyan A K, Karthik N. Study on drug utilization pattern of chronic renal failure patients in a tertiary care hospital. Internatinoal Journal of Pharmacy and Pharmaceutical Sciences. 2014; 6 (9): 482-484.

[2] Henok Getachew, Yewondwossen Tadesse, Workineh Shibeshi. Drug dosage adjustment in hospitalized patients with renal impairment at Tikur Anbessa specialized hospital, Addis Ababa, Ethiopia. BMC Nephrology. 2015; 16: 158.

[3] Rajiv Ahlawat, Sanjay D'cruz, Pramil Tiwari. Drug utilization pattern in chronic kidney disease patients at a tertiary care public teaching hospital: Evidence from a cross-sectional study. J Pharma Care Health. 2016; 3: 1.

[4] Marlies Karsch-Volk, Elisa Schmid. et al. Kidney function and clinical recommendations of drug dose adjustment in geriatric patients. BMC Geriatrics. 2013; 13: 92.

[5] Neeta J. Kanani, Vimesh R. Mistry, Sandeep J. Rathva. Drug utilization pattern in chronic kidney disease patients at a tertiary care teaching hospital. Innova Pharm Pharmacother. 2019; 7 (2): 31-36.

[6] Sindura P, Balaiah Sandyapakula, B Silvya Grace. et al. Assessment of drug prescribing pattern in patient of CKD along with CVD in tertiary care hospital. Int. J. Pharm. Sci. Rev. Res. 2018; 49 (2): 33-38.

[7] Sridhar Srimath Tirumala Konduru, Javvaji Naga Sai Kumar. et al. Assessment of drug use patterns and quality of life in haemodialysis patients. EJPMR. 2018; (6): 628-637.

[8] Latha Kamath, Hema N. G, Himamani S. A study of drug utilization pattern in patients of chronic kidney disease at a tertiary care hospital. IJBCP. 2019 Feb; 8 (2): 170-175.

[9] Soumya Sandra, Divya Agarwal, Sanjay Kumar, Sudhanshu Sekhar Mishra. A study on the drug utilization pattern in patients with chronic kidney disease with emphasis on antibiotics. J Integr Nephrol Androl. 2015; 2: 85-9.

[10] Paul Muntner, Amanda Anderson, Jeanne C, Zhen Chen, Virginia Ford, Gail M. et al. Hypertension awareness, treatment, and control in adults with ckd: results from the chronic renal insufficiency cohort (cric) study. Am J Kidney Dis. 2010 March; 55 (3): 441-445.

[11] P Anushman Abishek, Rajendra Panda, Rajashree Samal, Namitha M, Srikantha Mohanty. Drug utilization pattern and adverse events in patients with chronic kidney disease undergoing maintanence haemodialysis at a teritiary care hospital of odisha. Journal of Clinical and Diagnostic Research. 2017 Oct; 11 (10): 11-16.

[12] Rayane Saad, Souheli Hallit, Bahia Chahine. Evaluation of renal drug dosing adjustment in chronic kidney disease patients at two university hospitals in Lebanon. Pharmacy Practice. 2019 Jan-March; 17 (1): 1304.
[13] Abdulrahman M Alahdal, Ahmed A E. Evaluation of applying drug dose adjustment by physicians in patients with renal impairment. Saudi Pharmaceutical Journal. 2012; 20: 217-220.

[14] Aurelio C. M, Elena U Sanz, Carles I Navalon, Juan B. C Romero, Juan J Gascon Canovas. Influence of equation used to estimate the renal function in dosage potentially nephrotoxic drug. Eur J Hosp Pharm. 2015; 22: 23-27.

[15] Shivraj G, Prakash B D, Shruthi S K, Vinayak V H, Avinash A K M, Sonal N V. Markers of renal function. N Am J Med Sci. 2010 April; 2 (4): 170-173.

[16] Tom N. L. H, Jane E. C, Sophie L. S, Jacob Stevastos, Darren M. R. Clinical pharmacokinetics in kidney disease. Clin J Am Soc Nephrol. 2018 July 6; 13 (7): 1085-1095.

[17] Rowa Al- Ramahi. Medication prescribing patterns among chronic kidney disease patients in a hospital in malaysis. Saudi J Kidney Dis Transpl. 2012; 23 (2): 403-408.

[18] John W P, John M. A simple method to detect recovery of glomerular filtration rate following acute kidney injury. Biomed Research International. 2014; 55; 61-69.

[19] Beverly E, Liana D, Castel, Mathew McGinnis, Amy B, Rudolph C Cane Jr. et al. Economic and clinical outcomes resulting from the stage 4 chronic kidney disease case management quality improvement initiative. Prof Case Management. 2017; 22 (6): 291-298.

[20] Ayse L, Thomas C D, Mathew R W, Stephen L S, Robert H C, Laurence S M. Hepatology. 2014 April; 59 (4): 1532-1542.

[21] Leonie V M, Moerland, Adam F C, Jacobus B. Urinary kidney biomarkers for early detection of nephrotoxicity in clinical drug development. British Journal of Clinical Pharmacology. 2013; 10: 12-23.

[22] Gudrun B, Bengt R, Sven Erik Ricksten. Assessing glomerular filtration rate in critically ill patients with acute kidney injurytrue GFR versus urinary creatinine clearance and estimating equations. Critical Care. 2013; 17: R108.

[23] Anna T M, Steven F, Yoshitsugu O, Kamyar K Z. Preservation of residual kidney function in hemodialysis patients: reviving an old concept for contemporary practice. Kidney Int. 2016 Aug; 90 (2): 262-271.

[24] Stephan G, David H, Saber B, Alexander Z, Nuttha L, Ron Wald et al. Delayed versus early initiation of renal replacement therapy for severe acute kidney injury. Lancet. 2020; 395: 1506-1515.

[25] Min Zhan, Wendy L. St. Peter et al. Patterns of NSAIDs use and their association with other analgesic use in CKD. Clin J Am Soc Nephrol. 2017 Nov 7; 12 (11): 1778-1788.

[26] Mahboob Rahman, Dawei C, Harold IF, Alan S G, Jiang H, John W K. et al. Association between chronic kidney disease progression and cardiovascular disease: Results from the CRIC study. Am J Nephrol. 2014; 40 (5): 399-407.

[27] Manjula K T, Kristi E Yaffe, Chi-Yuan Hsu, Jingrong Yang, Stephen Sozio, Michael F. et al. Cognitive impairement and progression of CKD. Amj Kidney Dis. 2016 july; 68 (1): 77-83.

[28] Mierala D, Wei Yang, Qiang Pan, Lawrence A, Keith B, Jing Chen et al. Persistent high serum bicarbonate and the risk of heart failure in patients with chronic kidney disease. J Am Heart Assoc. 2015; 4: 144-159. 
[29] Elaine K U, Charles E, McCulloch, Eric V, Feng Li, Kirsten L J. Use of antihypertensive agents and association with risk of adverse outcomes in chronic kidney disease: focus on angiotensin converting enzyme inhibitors and angiotensin receptor blockers. J Am Heart Assoc. 2018; 7: 155-161.

[30] Jiang He, Michael S, Amanda A, Jason A R, Harold I F, Radhakrishna Reddy $\mathrm{K}$ et al. Risk factors for heart failure in patients with chronic kidney disease. J Am Heart Assoc. 2017; 6: 33-41.

[31] Tamara I, Cheryl A M Anderson, Mary B L, Dawei Xie, Orlando M G, Leigh K R. et al. Diuretics, calciuria and secondary hyperparathyroidism in the chronic renal insufficiency cohort (CRIC). Nephrol Dial Transplant (2011); 26: $1258-1265$.

[32] Jay Shah, Tejas K, Saurabh B, Rima B S, Sanjay D. Study of utilization pattern of anti-hypertensive drugs in hypertensive diabetic patients with or without reduced renal function at tertiary care teaching hospital. ijmsph. 2013; 2: 167-172.

[33] Samina S Khan, Waqar H K, Rekha A, Hocine T, Brian J. G. P, Annamaria T K. Health care utilization among patients with chronic kidney disease. Kidney International. 2002; 62: 229236. 